\title{
A Kinetic Model for Chemical Reactions without Barriers
}

Cite as: AIP Conference Proceedings 1084, 105 (2008); https://doi.org/10.1063/1.3076438

Published Online: 12 January 2009

Gilberto M. Kremer, and Ana Jacinta Soares

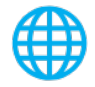

Lock-in Amplifiers Find out more today

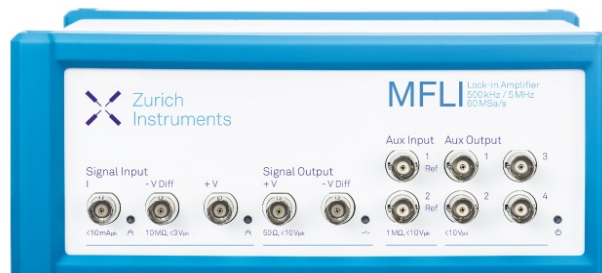

Zurich

Instruments 


\title{
A Kinetic Model for Chemical Reactions without Barriers
}

\author{
Gilberto M. Kremer* and Ana Jacinta Soares ${ }^{\dagger}$ \\ ${ }^{*}$ Departamento de Física, Universidade Federal do Paraná, Curitiba, Brazil \\ ${ }^{\dagger}$ Departamento de Matemática, Universidade do Minho, Braga, Portugal
}

\begin{abstract}
A system of coupled Boltzmann equations (BE) is here proposed for a binary gaseous mixture undergoing elastic and reactive collisions. Reactive cross sections without activation energy, i.e. without barriers, are adopted to model the chemical process, whereas differential cross sections of rigid spheres are assumed for elastic scattering. The possibility of a pair of molecules to collide through an elastic mechanism or a reactive process is described by means of probability coefficients which are introduced in the collision terms. The rate of reaction and temperature exchange rate are explicitly computed using the non-equilibrium solution of the BE obtained through the Chapman-Enskog method in a chemical regime such that the reactive process is in its initial stage. Spatially homogeneous solutions are examined for the number density of reactants and mixture temperature.
\end{abstract}

Keywords: Boltzmann equation, Chemical reactions

PACS: $51.10,+\mathrm{y}$ 47.70.Fw

\section{INTRODUCTION}

In the literature of the Boltzmann equation extended to chemically reacting gases, most research papers introduce reactive cross sections with activation energy whereas only few works consider reactive models for processes without activation energy [1]. On the other hand, chemical reactions without barriers have become an interesting topic in many engineering applications of reactive flows, in particular in combustion phenomena, but also in other fields such as astrophysics, organic chemistry, chemical physics and biophysics $[2,3]$. The modeling of reactions without barriers can help to interpret the related experimental data and provide some useful information about the kinetics of the reaction. Following this idea, a kinetic model of the Boltzmann equation is here proposed for a binary mixture undergoing elastic and reactive scattering. An appropriate model of reactive cross section without barriers can be found in the literature (see $[4,5]$ ) and it will correspond to the choice of the proposed non-barrier kinetic theory. At the microscopic scale, the key idea is to modify the collision terms of the Boltzmann equation, introducing probability coefficients that describe the possibility of a pair of molecules to collide through an elastic mechanism or a reactive process. This improvement accounts for the fact that a great number of reactive interactions corresponds to a small number of elastic collisions, and vice-versa. In this sense, the considered model represents a new approach, since almost all existent papers introduce reactive cross sections with activation energy and control the presence of both elastic and reactive collisions assuming rather large activation energies and a steric factor which reduce the number of reactive collisions.

In a chemical regime of slow reaction, the chemical non-equilibrium effects induced on the solution of the Boltzmann equation are also characterized using the Chapman-Enskog method and a second order Sonine polynomial expansion of the distributions. The chemical process is considered in its early stage so that reactive collisions among molecules of reaction products can be disregarded since the products of the reaction are in a small amount with respect to the reactants. Moreover, it was considered that the heat of reaction is a small quantity which can be neglected. At last, as an application of the thermodynamic model proposed here, spatially homogeneous field equations for reactant number density and mixture temperature are solved and the time evolution of the reactive system is examined.

\section{BOLTZMANN EQUATION AND MACROSCOPIC DESCRIPTION}

Consider a binary mixture of constituents $\alpha=A, B$ with binding energies $\varepsilon_{A}$ and $\varepsilon_{B}$ and equal molecular masses, $m_{A}=m_{B}=m$, undergoing a reversible reaction of type $\mathrm{A}+\mathrm{A} \rightleftharpoons \mathrm{B}+\mathrm{B}$. Gas molecules may participate in binary elastic collisions preserving momentum and kinetic energy,

$$
m \mathbf{c}_{\alpha}+m \mathbf{c}_{\beta}=m \mathbf{c}_{\alpha}^{\prime}+m \mathbf{c}_{\beta}^{\prime}, \quad \frac{1}{2} m c_{\alpha}^{2}+\frac{1}{2} m c_{\beta}^{2}=\frac{1}{2} m c_{\alpha}^{\prime 2}+\frac{1}{2} m c_{\beta}^{\prime 2},
$$

CP1084, Rarefied Gas Dynamics: $26^{\text {th }}$ International Symposium, edited by T. Abe (C) 2009 American Institute of Physics 978-0-7354-0615-5/09/\$25.00 
as well as in binary reactive interactions preserving momentum and total energy,

$$
m \mathbf{c}_{A}+m \mathbf{c}_{A_{1}}=m \mathbf{c}_{B}+m \mathbf{c}_{B_{1}}, \quad \varepsilon_{A}+\frac{1}{2} m c_{A}^{2}+\varepsilon_{A}+\frac{1}{2} m c_{A_{1}}^{2}=\varepsilon_{B}+\frac{1}{2} m c_{B}^{2}+\varepsilon_{B}+\frac{1}{2} m c_{B_{1}}^{2} .
$$

Above, $\left(\mathbf{c}_{\alpha}, \mathbf{c}_{\beta}\right)$ and $\left(\mathbf{c}_{\alpha}^{\prime}, \mathbf{c}_{\beta}^{\prime}\right)$ denote pre and post collisional velocities, whereas $\left(\mathbf{c}_{A}, \mathbf{c}_{A_{1}}\right)$ and $\left(\mathbf{c}_{B}, \mathbf{c}_{B_{1}}\right)$ refer to the the velocities of reactants and products of the forward reaction, respectively. Sub-index 1 is used to distinguish two molecules of the same species. The relative velocities of the reactants and products is given by $\mathbf{g}_{A}=\mathbf{c}_{A_{1}}-\mathbf{c}_{A}$ and $\mathbf{g}_{B}=\mathbf{c}_{B_{1}}-\mathbf{c}_{B}$, and the reaction heat is defined as $Q_{R}=2\left(\varepsilon_{A}-\varepsilon_{B}\right)$. A pertinent choice of reactive cross sections to describe chemical reactions without a barrier should not consider an activation energy. Accordingly, assuming an attractive potential energy of the form $V(r)=-K / r^{\mathrm{n}}$, the following reactive cross sections model is adopted (see Refs. $[4,5])$,

$$
\sigma_{\alpha}^{*}=\frac{\mathrm{d}_{\mathrm{r}}^{2}}{4}\left(\frac{m g_{\alpha}^{2}}{4 k T_{0}}\right)^{\mathrm{n}-1 / 2}
$$

where $T_{0}$ is a characteristic temperature, $\mathrm{d}_{\mathrm{r}}$ a reactive collision diameter and the exponent $\mathrm{n}$ may range from $-3 / 2$ to $1 / 2$, with the particular values $1 / 2,0$ and $1 / 6$ standing for hard-spheres reactions, ion-molecule reactions and reactions of neutral species, respectively. For what concerns the differential cross sections of elastic encounters, the simple model of rigid spheres is adopted, namely $\sigma_{\alpha \beta}=\mathrm{d}^{2} / 4$, where $\mathrm{d}$ is the molecular diameter.

The Boltzmann equation proposed here for the considered mixture contains, as usual, distinct contributions for elastic collisions and reactive interactions, but now suitable probability coefficients $0 \leq \chi_{A}, \chi_{B} \leq 1$ are introduced in order to account for the possibility of a pair of molecules interact through an elastic collision or a reactive process. In absence of external body forces, the $\mathrm{BE}$ for $f_{\alpha}$ is written then in the form

$$
\begin{array}{r}
\frac{\partial f_{\alpha}}{\partial t}+c_{i}^{\alpha} \frac{\partial f_{\alpha}}{\partial x_{i}}=\sum_{\beta=A}^{B}\left(1-\chi_{\beta} \delta_{\alpha \beta}\right) \int\left[f_{\alpha}^{\prime} f_{\beta}^{\prime}-f_{\alpha} f_{\beta}\right] g_{\beta \alpha} \sigma_{\alpha \beta} d \Omega_{\alpha \beta} d \mathbf{c}_{\beta} \\
+\int\left[\chi_{\beta} f_{\beta} f_{\beta_{1}} \sigma_{\beta}^{\star} \frac{g_{\beta}^{2}}{g_{\alpha}^{2}}-\chi_{\alpha} f_{\alpha} f_{\alpha_{1}} \sigma_{\alpha}^{\star}\right] g_{\alpha} d \Omega_{\beta} d \mathbf{c}_{\alpha_{1}},
\end{array}
$$

The first term on the r.h.s. of (4) refers to elastic collisions and the second one to chemical interactions where one has to take $\alpha \neq \beta=A, B$. Elastic contributions referred to $A A$ and $B B$ encounters are affected by a reduced factor, namely $1-\chi_{\alpha}, \alpha=A, B$, which is compensated by the corresponding factor $\chi_{\alpha}$ relative to reactive encounters among $A A$ and $B B$ molecules, respectively.

The macroscopic picture of the mixture can be described by the number densities $n_{\alpha}$ and internal energy densities $\rho_{\alpha} e_{\alpha}$ of both constituents, together with the mixture velocity $v_{i}$, given by

$$
n_{\alpha}=\int f_{\alpha} d \mathbf{c}_{\alpha}=\frac{\rho_{\alpha}}{m}, \quad v_{i}=\frac{1}{\rho} \sum_{\alpha=A}^{B} \int m c_{i}^{\alpha} f_{\alpha} d \mathbf{c}_{\alpha}, \quad \rho_{\alpha} e_{\alpha}=\int \frac{m}{2} \xi_{\alpha}^{2} f_{\alpha} d \mathbf{c}_{\alpha}
$$

with $\xi_{i}^{\alpha}=c_{i}^{\alpha}-v_{i}$ the peculiar velocity of constituent $\alpha$, and $n=\sum_{\alpha=A}^{B} n_{\alpha}, \rho=\sum_{\alpha=A}^{B} \rho_{\alpha}$. Each constituent is assumed to have the same temperature $T$ which is the mixture's temperature, so that its internal energy density becomes $\rho_{\alpha} e_{\alpha}=3 n_{\alpha} k T / 2$. By rather standard arguments, the balance equations for the fields defined in expressions (5) are obtained from Eq. (4), in the form

$$
\begin{gathered}
\frac{\partial n_{\alpha}}{\partial t}+\frac{\partial}{\partial x_{i}}\left(n_{\alpha} u_{i}^{\alpha}+n_{\alpha} v_{i}\right)=\tau_{\alpha} \\
\frac{\partial \rho v_{i}}{\partial t}+\frac{\partial}{\partial x_{j}}\left(p_{i j}+\rho v_{i} v_{j}\right)=0 \\
\frac{3}{2} \frac{\partial n_{\alpha} k T}{\partial t}+\frac{\partial}{\partial x_{i}}\left(q_{i}^{\alpha}+\frac{3}{2} n_{\alpha} k T v_{i}\right)-\frac{\rho_{\alpha}}{\rho} u_{i}^{\alpha} \frac{\partial p_{i j}}{\partial x_{j}}+p_{i j}^{\alpha} \frac{\partial v_{j}}{\partial x_{i}}=\zeta_{\alpha} .
\end{gathered}
$$


Above, $\tau_{\alpha}$ is the rate of reaction and $\zeta_{\alpha}$ the production term of internal energy density of constituent $\alpha$, given by

$$
\begin{aligned}
\tau_{\alpha}= & \int\left[\chi_{\beta} f_{\beta} f_{\beta_{1}} \sigma_{\beta}^{\star} \frac{g_{\beta}^{2}}{g_{\alpha}^{2}}-\chi_{\alpha} f_{\alpha} f_{\alpha_{1}} \sigma_{\alpha}^{\star}\right] g_{\alpha} d \Omega_{\beta} d \mathbf{c}_{\alpha_{1}} d \mathbf{c}_{\alpha}, \quad \text { with } \tau_{B}=-\tau_{A}, \\
\zeta_{\alpha}= & \sum_{\beta=A}^{B}\left(1-\chi_{\beta} \delta_{\alpha \beta}\right) \int \frac{1}{2} m\left(\xi_{\alpha}^{\prime 2}-\xi_{\alpha}^{2}\right) f_{\alpha} f_{\beta} g_{\beta \alpha} \sigma_{\beta \alpha} d \Omega_{\beta \alpha} d \mathbf{c}_{\beta} d \mathbf{c}_{\alpha} \\
& +\int \frac{m}{4}\left(\xi_{\alpha}^{2}+\xi_{\alpha_{1}}^{2}\right)\left[\chi_{\beta} f_{\beta} f_{\beta_{1}} \sigma_{\beta}^{\star} \frac{g_{\beta}^{2}}{g_{\alpha}^{2}}-\chi \alpha f_{\alpha} f_{\alpha_{1}} \sigma_{\alpha}^{\star}\right] g_{\alpha} d \Omega_{\beta} d \mathbf{c}_{\alpha_{1}} d \mathbf{c}_{\alpha} .
\end{aligned}
$$

Moreover, $u_{i}^{\alpha}, q_{i}^{\alpha}$ and $p_{i j}^{\alpha}$ are the diffusion velocity, heat flux and pressure tensor of each constituent, defined by

$$
u_{i}^{\alpha}=\frac{1}{n_{\alpha}} \int \xi_{i}^{\alpha} f_{\alpha} d \mathbf{c}_{\alpha}, \quad q_{i}^{\alpha}=\int \frac{1}{2} m \xi_{\alpha}^{2} \xi_{i}^{\alpha} f_{\alpha} d \mathbf{c}_{\alpha}, \quad p_{i j}^{\alpha}=\int m \xi_{i}^{\alpha} \xi_{j}^{\alpha} f_{\alpha} d \mathbf{c}_{\alpha}
$$

with

$$
\sum_{\alpha=A}^{B} \rho_{\alpha} u_{i}^{\alpha}=0, \quad q_{i}=\sum_{\alpha=A}^{B} q_{i}^{\alpha}, \quad p_{i j}=\sum_{\alpha=A}^{B} p_{i j}^{\alpha}
$$

\section{THE NON-EQUILIBRIUM DISTRIBUTION FUNCTION}

The deviations induced by the chemical reaction on the distribution functions can be explicitly computed using the Chapman-Enskog method [9] with Sonine polynomial representation of the distributions. Assuming a chemical regime of slow processes, for which the reaction is close to its initial stage, reactive collisions are less frequent than elastic encounters and the chemical relaxation time is larger than the elastic one. This means that reactive collision terms of the Boltzmann Eqs. (4) and material time derivatives, $\mathscr{D}=(\partial / \partial t)+v_{i} \partial / \partial x_{i}$, are of the same order, whereas the gradients of the fields are of successive order. This means that the Boltzmann equation (4) can be written as

$$
\begin{aligned}
\mathscr{D} f_{\alpha}+\lambda \xi_{i}^{\alpha} \frac{\partial f_{\alpha}}{\partial x_{i}}-\int\left[\chi_{\beta} f_{\beta} f_{\beta_{1}} \sigma_{\beta}^{\star} \frac{g_{\beta}^{2}}{g_{\alpha}^{2}}-\chi_{\alpha} f_{\alpha} f_{\alpha_{1}} \sigma_{\alpha}^{\star}\right] g_{\alpha} d \Omega_{\beta} d \mathbf{c}_{\alpha_{1}} \\
=\frac{1}{\lambda} \sum_{\beta=A}^{B}\left(1-\chi_{\beta} \delta_{\alpha \beta}\right) \int\left[f_{\alpha}^{\prime} f_{\beta}^{\prime}-f_{\alpha} f_{\beta}\right] g_{\beta \alpha} \sigma_{\alpha \beta} d \Omega_{\alpha \beta} d \mathbf{c}_{\beta}
\end{aligned}
$$

where $\lambda$ is a formal parameter of the order of the Knudsen number [9]. Proceeding with the insertion of the expansions

$$
f_{\alpha}=f_{\alpha}^{M}+\lambda f_{\alpha}^{(0)}+\lambda^{2} f_{\alpha}^{(1)}+\ldots \quad \text { and } \quad \mathscr{D}=\mathscr{D}^{(0)}+\lambda \mathscr{D}^{(1)}+\lambda^{2} \mathscr{D}^{(2)}+\ldots
$$

into the Boltzmann Eqs. (11) and equating equal powers of $\lambda$, one obtains the integral equations for $f_{\alpha}^{M}$ and $f_{\alpha}^{(0)}$ in the form,

$$
\begin{gathered}
\sum_{\beta=A}^{B}\left(1-\chi_{\beta} \delta_{\alpha \beta}\right) \int\left[f_{\alpha}^{M \prime} f_{\beta}^{M \prime}-f_{\alpha}^{M} f_{\beta}^{M}\right] g_{\beta \alpha} \sigma_{\alpha \beta} d \Omega_{\alpha \beta} d \mathbf{c}_{\beta}=0 \\
\mathscr{D}^{(0)} f_{\alpha}^{M}-\int\left[\chi_{\beta} f_{\beta}^{M} f_{\beta_{1}}^{M} \sigma_{\beta}^{\star} \frac{g_{\beta}^{2}}{g_{\alpha}^{2}}-\chi_{\alpha} f_{\alpha}^{M} f_{\alpha_{1}}^{M} \sigma_{\alpha}^{\star}\right] g_{\alpha} d \Omega_{\beta} d \mathbf{c}_{\alpha_{1}} \\
=\sum_{\beta=A}^{B}\left(1-\chi_{\beta} \delta_{\alpha \beta}\right) \int\left[f_{\alpha}^{(0) \prime} f_{\beta}^{M \prime}+f_{\alpha}^{M \prime} f_{\beta}^{(0) \prime}-f_{\alpha}^{(0)} f_{\beta}^{M}-f_{\alpha}^{M} f_{\beta}^{(0)}\right] g_{\beta \alpha} \sigma_{\alpha \beta} d \Omega_{\alpha \beta} d \mathbf{c}_{\beta} .
\end{gathered}
$$

The solution of the integral equation (13) is the Maxwellian distribution function, given by

$$
f_{\alpha}^{M}=n_{\alpha}\left(\frac{m}{2 \pi k T}\right)^{\frac{3}{2}} e^{-\frac{m \xi_{\alpha}^{2}}{2 k T}}
$$


with partial number densities $n_{\alpha}$ completely uncorrelated, since no chemical equilibrium condition is involved.

Concerning Eq. (14), since the field gradients are absent, one admits that its solution is a small deviation from the Maxwellian distribution, expressed in terms of Sonine polynomials as

$$
f_{\alpha}^{(0)}=f_{\alpha}^{M}\left[a_{1}^{\alpha}\left(\frac{3}{2}-\frac{m \xi_{\alpha}^{2}}{2 k T}\right)+a_{2}^{\alpha}\left(\frac{15}{8}-\frac{5 m \xi_{\alpha}^{2}}{4 k T}+\frac{m^{2} \xi_{\alpha}^{4}}{8 k^{2} T^{2}}\right)\right]
$$

where $a_{1}^{\alpha}$ and $a_{2}^{\alpha}$ are scalar coefficients to be determined. First, the insertion of distributions (16) into definition (5) of the internal energy density and integration of the resulting equation lead to $a_{1}^{A}=a_{1}^{B}=0$. Afterwards, the balance Eqs. (6-8) for an Eulerian mixture where $u_{i}^{\alpha}=0, p_{i j}=p \delta_{i j}$ and $q_{i}=0$ are used to eliminate the material derivatives in Eq. (14). The successive multiplication of the resulting equation by $m$ and $\left(3 / 2-m \xi_{\alpha}^{2} / 2 k T\right)$ and further integration over $\mathbf{c}_{\alpha}$ lead to identities, whereas the multiplication by $\left(15 / 8-5 m \xi_{\alpha}^{2} / 4 k T+m^{2} \xi_{\alpha}^{4} / 8 k^{2} T^{2}\right)$ and integration give the following system of coupled equations for $a_{2}^{A}$ and $a_{2}^{B}$

$$
-v_{\alpha} \frac{\chi_{A} n_{A}^{2}}{2}\left(\frac{T}{T_{0}}\right)^{\mathrm{n}-\frac{1}{2}} \Gamma\left(\mathrm{n}+\frac{3}{2}\right) \mathrm{n}(\mathrm{n}-1)\left(\frac{\mathrm{d}_{\mathbf{r}}}{\mathrm{d}}\right)^{2}=-4\left(1-\chi_{\alpha}\right) n_{\alpha}^{2} a_{2}^{\alpha}+\frac{n_{\alpha} n_{\beta}}{4}\left(15 a_{2}^{\beta}-31 a_{2}^{\alpha}\right), \quad \alpha \neq \beta=A, B
$$

where $v_{A}=-v_{B}=-1$ are stoichiometric coefficients. For the purpose of the present study, it was considered that in the early stage of the reaction the probability of a reactive collision between $B$ molecules is negligible so that $\chi_{B} \approx 0$. Furthermore, the heat of reaction is a small quantity so that it can be neglected. These assumptions have been taken into account to deduce Eqs. (17). More details about the approximating procedure here employed are reported in a paper in preparation, concerning the kinetic theory of chemical reactions without barriers (see Ref. [10]).

From the system of equations (17) one obtains

$$
\begin{gathered}
a_{2}^{A}=\frac{2 \chi_{A} x_{A}}{15 x_{A}^{2} \chi_{A}+16 x_{A} \chi_{A}-31}\left(\frac{T}{T_{0}}\right)^{\mathrm{n}_{-\frac{1}{2}}} \Gamma\left(\mathrm{n}+\frac{3}{2}\right) \mathrm{n}(\mathrm{n}-1)\left(\frac{\mathrm{d}_{\mathbf{r}}}{\mathrm{d}}\right)^{2}, \\
a_{2}^{B}=\frac{x_{A}\left(x_{A} \chi_{A}-1\right)}{1-x_{A}} a_{2}^{A},
\end{gathered}
$$

where $x_{A}=n_{A} / n$ and $x_{B}=1-x_{A}$ represent the molar fraction of the constituents.
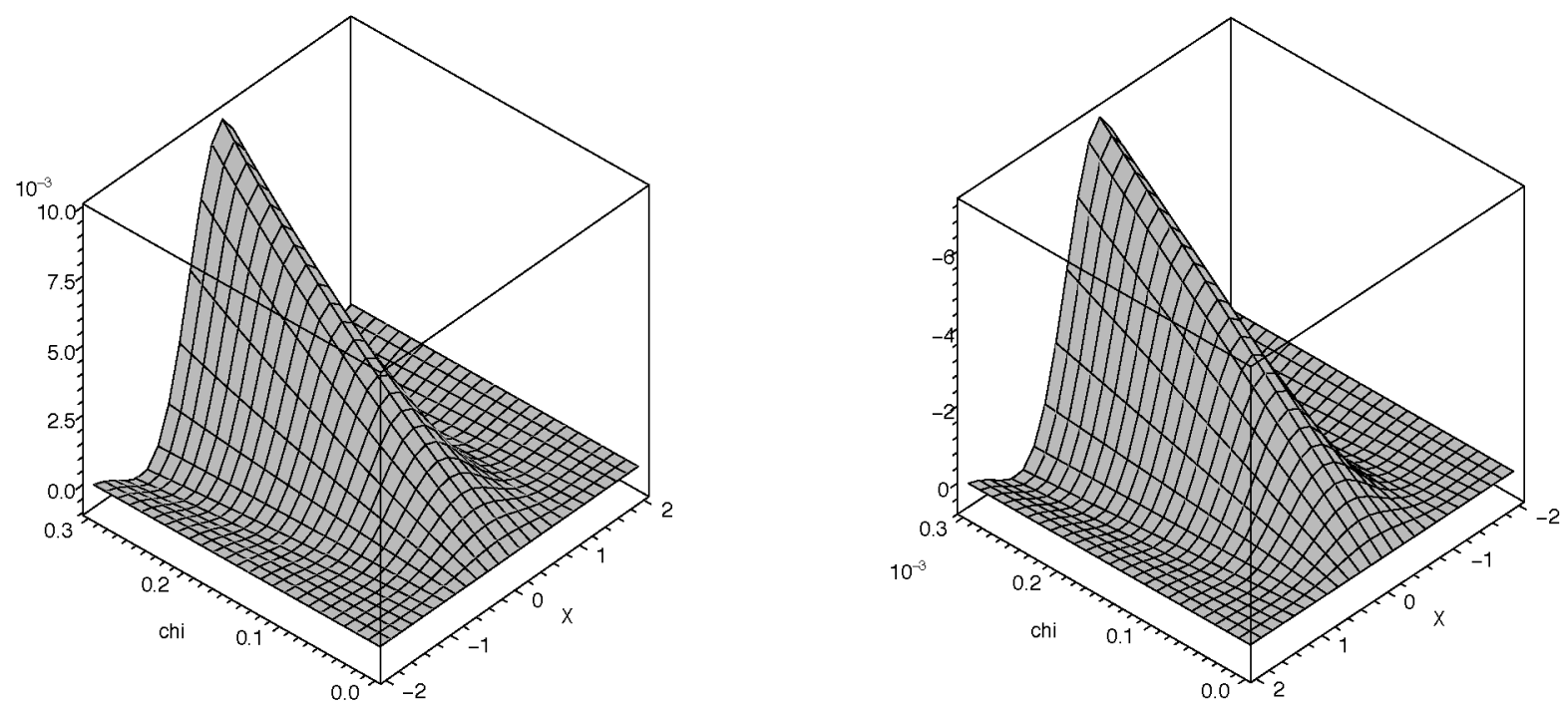

FIGURE 1. Deviations of the non-equilibrium distribution function with respect to the Maxwellian distribution, versus $X$ and chi $\equiv \chi_{A}$, for $x_{A}=0.9$. Left frame: $F_{A}$; right frame: $F_{B}$. 
All the representative effects induced on the non-equilibrium distribution function are illustrated in Fig. 1, where the deviations with respect to the Maxwellian distribution function, namely

$$
F_{\alpha} \equiv \frac{1}{n}\left(\frac{2 \pi k T}{m}\right)^{3 / 2} f_{\alpha}^{(0)}=x_{\alpha} e^{-X^{2}} a_{2}^{\alpha}\left(\frac{15}{8}-\frac{5}{2} X^{2}+\frac{1}{2} X^{4}\right), \quad \alpha=A, B
$$

are plotted versus chi $=\chi_{A}$ and $X=\sqrt{m \xi_{A}^{2} / 2 k T}$, when $\mathrm{d}_{\mathrm{r}}=\mathrm{d}$ and $x_{A}=0.9$ for the case of hard-spheres reactions without barrriers, i.e., for $n=1 / 2$. The left frame refers to the Maxwellian deviation for the reactants whereas the right frame to the corresponding deviations for the products. One can infer from the figures that the modulus of deviation from a Maxwellian distribution function becomes larger when the probability of reactive encounters among the reactants increases. Furthermore, the deviation is positive for the reactants and negative for the products, which means that the non equilibrium distribution is larger than the Maxwellian one for the reactants and smaller for the products.

Note that for $\chi_{A}=0$ the distribution is Maxwellian and all collisions are elastic, i.e., no reactive encounter occurs.

\section{A SPATIALLY UNIFORM PROBLEM}

From the knowledge of the non-equilibrium distribution function previously obtained, it is now possible to evaluate the reaction rate and internal energy exchange rate of the reactants. In fact, it is enough to insert expressions (16) into definitions (9) and (10) and performing the corresponding integrations. One obtains

$$
\begin{gathered}
\tau_{A}=-\sqrt{\frac{\pi k T}{m}}\left(\frac{T}{T_{0}}\right)^{\mathrm{n}-\frac{1}{2}} \Gamma\left(\mathrm{n}+\frac{3}{2}\right) \chi_{A} n_{A}^{2} \mathrm{~d}_{\mathbf{r}}^{2}\left[4+\mathrm{n}(\mathbf{n}-1) a_{2}^{A}\right], \\
\zeta_{A}=-k T \sqrt{\frac{\pi k T}{m}}\left\{n_{A} n_{B} \mathrm{~d}^{2}\left(a_{2}^{A}-a_{2}^{B}\right)+\frac{1}{2}\left(\frac{T}{T_{0}}\right)^{\mathrm{n}-\frac{1}{2}} \Gamma\left(\mathrm{n}+\frac{3}{2}\right) \chi_{A} n_{A}^{2} \mathrm{~d}_{\mathbf{r}}^{2}\left[12+4 \mathrm{n}+\mathrm{n}\left(5+4 \mathrm{n}+\mathrm{n}^{2}\right) a_{2}^{A}\right]\right\},
\end{gathered}
$$

with $a_{2}^{A}$ and $a_{2}^{B}$ given by expressions (18) and (19), respectively.

In the particular case of chemical reactions of ion-molecule type $(n=0)$, the effects related to the coefficients $a_{2}^{\alpha}$ disappear from the production terms (21) and (22), and also from the non-equilibrium deviations $f_{\alpha}^{(0)}$.

From the balance equations (6) and (8), with the production terms (21) and (22) referred to the present case, one obtains the spatially uniform evolution equations for particle number density and internal energy density of the reactants,

$$
\frac{d x_{A}}{d \mathrm{t}}=-\frac{\sqrt{\pi}}{2} \chi_{A} x_{A}^{2} \quad \text { and } \quad \frac{3}{2} k \frac{d\left(x_{A} T\right)}{d \mathrm{t}}=-\frac{3}{2} k T \frac{\sqrt{\pi}}{2} \chi_{A} x_{A}^{2}
$$

with

$$
\mathrm{t}=\frac{t}{t_{c}}, \quad t_{c}=\frac{1}{4 n_{0} \mathrm{~d}^{2}} \sqrt{\frac{m}{\pi k T_{0}}}
$$

where $t_{c}$ is the mean free time of elastic collisions, and $n_{0}, T_{0}$ denote the number density and temperature of the mixture in a reference initial state. Moreover the collision diameters have been considered equal to each other, i.e. $d_{r}=d$.

The solution of the differential equations (23) is given by

$$
x_{A}=\frac{x_{A}^{0}}{\frac{\sqrt{\pi}}{2} x_{A}^{0} \chi_{A} \mathbf{t}+1}, \quad T=T_{0} .
$$

From the above equations, one concludes that the temperature of the mixture remains constant while the reactant concentration decreases with time and that the decay is more pronounced when the probability $\chi_{A}$ of reactive encounters increases. This last result can be observed in Figure 2 where the ratio $x_{A} / x_{A}^{0}$ is represented as function of $\mathrm{t}$ and $\mathrm{chi}=\chi_{\mathrm{A}}$ for $x_{A}^{0}=0.9$. 


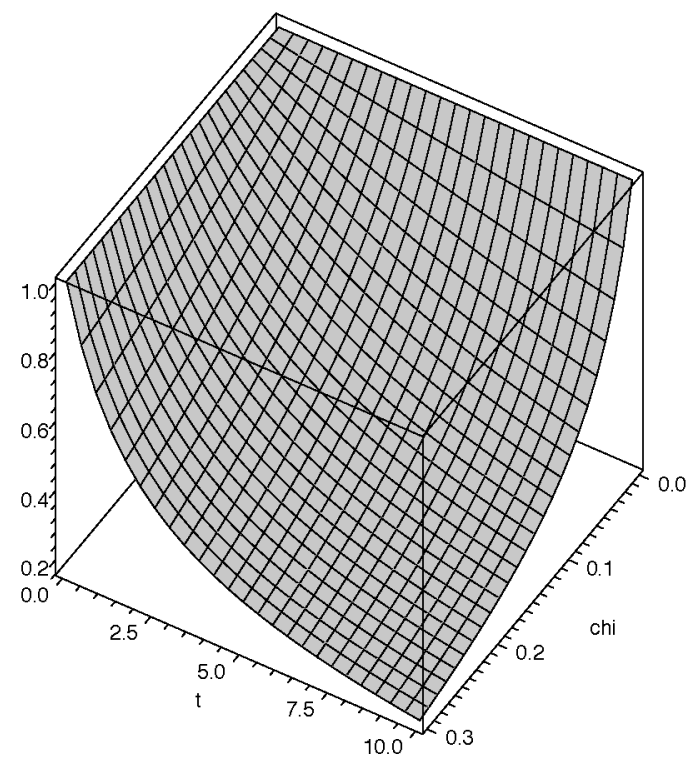

FIGURE 2. Ratio $x_{A} / x_{A}^{0}$ as function of $\mathrm{t}$ and $\operatorname{chi}=\chi_{A}$ for $x_{A}^{0}=0.9$.

\section{ACKNOWLEDGMENTS}

The paper is partially supported by Brazilian Research Council (CNPq), by Minho University Mathematics Centre (CMAT-FCT) and by Project FCT - PTDC/MAT/68615/2006. G.M.K. gratefully acknowledges the support and hospitality of the Minho University Mathematics Centre.

\section{REFERENCES}

1. B. D. Shizgal, A. Chikhaoui, Physica A, 365,317-332 (2006).

2. J. P. Hessler, J. Chem . Phys. 111, 4068-4076 (1999).

3. J. P. Guthrie, V. Pitchko, J. Phys. Org. Chem. 17, 548-559 (2004).

4. W. Stiller, Arrhenius Equation and Non-Equilibrium Kinetics, BSB Teubner Text, Leipzig, 1989.

5. M. Brouard, Reaction Dynamics, Oxford University Press, Oxford, 1998.

6. I. Prigogine, M. Mahieu, Physica 16, 51-64 (1950).

7. G. M. Kremer, A. J. Soares, J. Stat. Mech., P12003, 1-16 (2007).

8. I. Prigogine, R. Defay, Chemical Thermodynamics (Longman, London) 1973.

9. S. Chapman, T. G. Cowling, The mathematical theory of non-uniform gases, Cambridge University Press, Cambridge 1970.

10. G. M. Alves, G. M. Kremer, A. J. Soares, Kinetic theory of chemical reactions without barriers, manuscript in preparation. 